\title{
IPOMOEA DIEGOAE (CONVOLVULACEAE), UNA NUEVA ESPECIE PARA GUERRERO, MÉXICO
}

\author{
José Manuel Castro Lara
}

Laboratorio de Plantas Vasculares, Departamento de Biología, Facultad de Ciencias, Universidad Nacional Autónoma de México, Ciudad Universitaria, Coyoacán 04510 México, D.F.

\section{RESUMEN}

Se describe Ipomoea diegoae sp. nov., una especie endémica procedente del municipio de Iguala de la Independencia en el estado de Guerrero. El nuevo taxon se ubica en la sección Leptocallis y está relacionado con I. ternifolia Cav., de la cual difiere en el tamaño de las hojas, número de segmentos foliares y tamaño de la corola.

Palabras clave: Convolvulaceae, Guerrero, Ipomoea, México.

\begin{abstract}
Ipomoea diegoae sp. nov., an endemic species from the municipality of Iguala de la Independencia in the state of Guerrero, is described and illustrated. The species belongs to Ipomoea section Leptocallis and is closely related to I. ternifolia Cav., from which it differs in leaf size, quantity of leaf segments and size of the corolla.
\end{abstract}

Key words: Convolvulaceae, Guerrero, Ipomoea, Mexico.

\section{INTRODUCCIÓN}

La característica más distintiva de las plantas que componen la sección Leptocallis del género Ipomoea es la forma pedatisecta de las hojas (McDonald, 1995), carácter que algunos autores plasman en el nombre de la serie Pedatisectae (Austin, 1979, 1980). Son plantas relativamente pequeñas, no exceden de los $2 \mathrm{~m}$ de largo, poseen una estructura delicada y prefieren ambientes secos.

El número de especies que forman esta sección ha variado de acuerdo con el criterio de diferentes autores, así House (1908) reconoce 18, Matuda (1963) 
incluye solamente 14 y McDonald (1995) reduce el número de taxa a ocho. Se considera a México el lugar de origen de este grupo, aunque dos especies se distribuyen hasta América del Sur.

Al realizar la revisión del material colectado del género Ipomoea (Convolvulaceae) para el proyecto Flora de Guerrero, se encontraron algunos ejemplares pertenecientes a Ipomoea sección Leptocallis (G.Don) J.A. McDonald. Por sus características particulares no fue posible ubicarlos en una especie conocida, por lo que se propone como:

Ipomoea diegoae M.C.Lara sp. nov. Fig. 1.

Herba volubilis Ipomoeae ternifoliae affinis. Folia petiolata; lamina 0.9-8.6 cm longa, 1.7-9.8 cm lata, membranacea, glabra, palmatim dissecta cum 14-16 segmentis; segmenta ensiformia vel filiformia, interna longiora, 1-6.1 cm longa, 0.3-2.8 mm lata. Sepala inaequalia, externa 7.9-8.7 mm longa, 2-2.3 mm lata, interna 9-14 mm longa, 3-4.6 mm lata, elliptico-lanceolata, integra, nervio medio atroviridi, albo-scariosa subhyalina margine, obtusa mucronulata apice. Corollae infundibuliformes, 4.3-7.2 cm longae, roseae dilutae, purpureae pallidae in sicco, plicae extus viridulae; tubus purpureus interius, $0.9-2.2 \mathrm{~cm}$ longus, $4-6 \mathrm{~mm}$ latus, fauces $0.6-1.2 \mathrm{~cm}$ lata, interius purpurea; limbus roseus 3.9-6.7 cm latus. Stamina inaequalia; filamenta trichomatibus glandulosis base et sparsim omnino longitudine instructa, rosea intense ad vinosa saltem ad faucem.

Hierba anual escandente o postrada, de 0.9 a $1 \mathrm{~m}$ de altura. Raíz fibrosa. Tallo rollizo contorto, verde, la base de color pardo en la madurez, liso, glabro, de 0.9 a $1.7 \mathrm{~mm}$ de diámetro, entrenudos de 2.1 a $20.7 \mathrm{~cm}$ de largo. Hojas pecioladas, láminas suborbiculares u orbiculares en contorno general, base subcordada a cordada, profundamente palmatisectas, de 0.9 a $8.6 \mathrm{~cm}$ de largo y de 1.7 a $9.8 \mathrm{~cm}$ de ancho, con 14 a 16 segmentos foliares, los interiores más largos que los exteriores, ensiformes o filiformes, de 1 a $6.1 \mathrm{~cm}$ de largo y de 0.3 a $2.8 \mathrm{~mm}$ de ancho, membranáceos, glabros, de ápice agudo y mucronulado, base atenuada, márgenes enteros, de color verde en ambas superficies; envés blanco punteado con una vena central prominente en los segmentos de las hojas maduras, venación palmada; pecíolos rollizos, verdes, de color pardo al secarse, de 0.3 a $3.6 \mathrm{~cm}$ de largo y de 0.5 a $0.9 \mathrm{~mm}$ de ancho, lisos, glabros, con hojas reducidas en la base. Inflorescencia en monocasios o dicasios, a veces flores solitarias; pedúnculo primario erecto, rollizo, verde, de color pajizo al secarse, glabro, de 4.8 a $13.1 \mathrm{~cm}$ de largo, y de 1 a $1.3 \mathrm{~mm}$ de ancho; pedúnculo secundario similar al anterior, de $2.1 \mathrm{a} 6.3 \mathrm{~cm}$ de largo y de 1 a $1.3 \mathrm{~mm}$ de ancho; pedicelos erectos, rollizos, de 1.3 a $2 \mathrm{~cm}$ de 


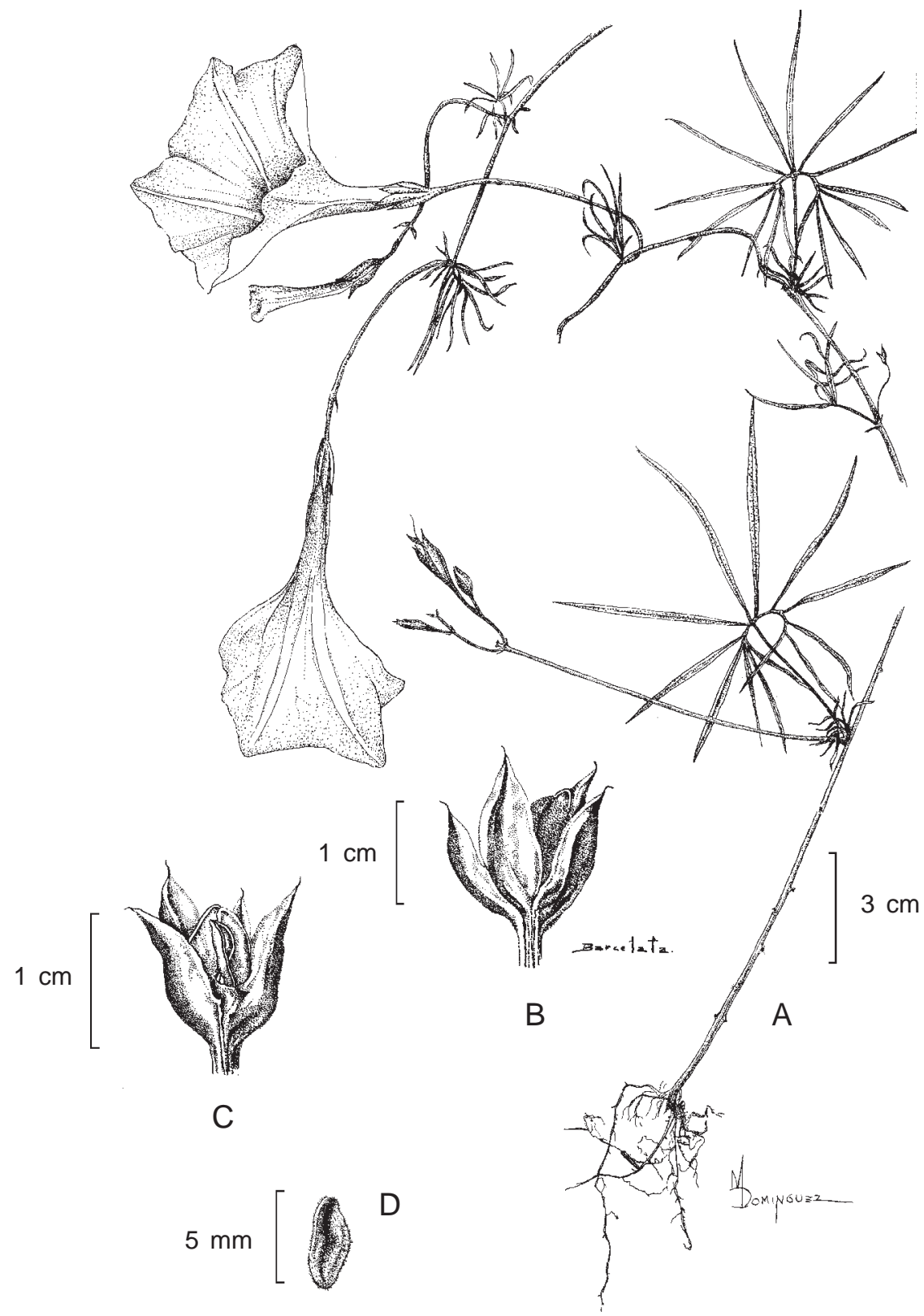

Fig. 1. Ipomoea diegoae, A. Rama con raíz, hojas y flores; B. Fruto mostrando detalle de los sépalos; C. Detalle del fruto maduro, donde se ha retirado un sépalo para mostrar las valvas de la cápsula; D. Semilla. Basado en M. Castro 40. 
largo y de 1.4 a $1.7 \mathrm{~mm}$ de ancho, verdes, de color pajizo al secarse, sulcados, glabros, con brácteas opuestas, persistentes, herbáceas, tripartidas, a veces monofilas, de aspecto similar a los sépalos, ovado-lanceoladas, de 1 a $2.2 \mathrm{~mm}$ de largo y de 0.4 a $0.9 \mathrm{~mm}$ de ancho, verdes oscuras, con un nervio central prominente, muriculadas, ápice acuminado, mucronulado, bordes enteros, escariosos; sépalos desiguales, los exteriores de 7.9 a $8.7 \mathrm{~mm}$ de largo y de 2 a $2.3 \mathrm{~mm}$ de ancho, los interiores de 9 a $14 \mathrm{~mm}$ de largo y de 3 a $4.6 \mathrm{~mm}$ de ancho, elíptico lanceolados, nervio central verde oscuro, bordes enteros, blanco escariosos, subhialinos, ápice obtuso, mucronulados; corola infundibuliforme, de 4.3 a $7.2 \mathrm{~cm}$ de largo, de color rosa claro, morado claro al secarse, las 5 líneas radiadas verdosas exteriormente en fresco, tubo morado en el interior, de 0.9 a $2.2 \mathrm{~cm}$ de largo y de 4 a $6 \mathrm{~mm}$ de ancho, garganta de 0.6 a $1.2 \mathrm{~cm}$ de ancho, morada interiormente, limbo de color rosa, de 3.9 a $6.7 \mathrm{~cm}$ de ancho, estivación contorta induplicada; estambres desiguales, filamentos con pelos glandulares en la base, escasamente en toda su longitud, de color rosa fuerte a guinda al menos hasta la altura de la garganta, de 1 a $2.3 \mathrm{~cm}$ de largo, el estambre más largo excede en más del doble en longitud al más pequeño, anteras de 4 a $5 \mathrm{~mm}$ de largo y de $1.2 \mathrm{~mm}$ de ancho; estilo blanco, de color pajizo al secarse, de hasta $2.5 \mathrm{~cm}$ de largo; fruto una cápsula ovoidea, dehiscente en 4 valvas, de color pardo al secarse, de 0.9 a $1 \mathrm{~cm}$ de largo y de 5 a $7 \mathrm{~mm}$ de ancho, con 2 lóculos; semillas 4, a menudo 2 abortivas, elipsoides, de 5 a $5.8 \mathrm{~mm}$ de largo y de 2.2 a $2.6 \mathrm{~mm}$ de ancho, su superficie furfurácea, con pelillos plateados y dos manchas negras longitudinales.

Tipo: México, Guerrero: municipio de Iguala de la Independencia, a $8 \mathrm{~km}$ de Iguala sobre carretera 51 rumbo a Ciudad Altamirano, 4.XI.1989. M. Castro 40 (FCME). Isotipos (ENCB, MEXU).

Paratipos: misma localidad, 14.X.1999, M. Castro 179 (ENCB, FCME, IEB, MEXU); El Platanal 4 km al NE, desviación a Huitziltepec, municipio Eduardo Neri, S. Valencia 1034 (FCME); El Palmar $2 \mathrm{~km}$ al N, municipio Eduardo Neri. $J$. Jiménez y M. Luna 1060 (FCME); Barranca de Amoloncán, a 3 km de San Agustín Oapan rumbo a San Juan Tetelcingo, municipio Tepecoacuilco de Trujano, E. Domínguez, G. Hall y J. Amith 79 (FCME); Jilotepec, cañada de Las Pozas, municipio Xochihuehuetlán, E. Moreno 708 (FCME); Paraje Ocotepec cerro Xilotzin, Jilotepec, municipio Xochihuehuetlán, E. Moreno 878 (FCME).

I. diegoae prospera en elevaciones entre 500 y $1580 \mathrm{~m}$ s.n.m. formando parte del bosque tropical caducifolio y bosque de Quercus. 
Fenología: Se ha colectado con flor y fruto de septiembre a noviembre, aunque probablemente este período pueda extenderse hasta febrero, ya que hay ejemplares con flores que se han colectado en ese mes.

Hábitat: Ipomoea diegoae crece como parte de la vegetación secundaria a la orilla de caminos o parcelas de cultivo en el valle de Iguala, Guerrero; la localidad tipo está situada a $18^{\circ} 22^{\prime \prime} \mathrm{N}$ y $99^{\circ} 33^{\prime \prime} \mathrm{W}$, a $730 \mathrm{~m}$ de altitud, con clima del tipo $\mathrm{Aw}_{\mathrm{o}}(\mathrm{w})\left(\mathrm{i}^{\prime}\right) \mathrm{g}$, cálido subhúmedo con lluvias en verano, la temperatura media anual es de $26^{\circ} \mathrm{C}$, la precipitación es de $1049 \mathrm{~mm}$ en promedio al año. Los suelos son derivados de depósitos aluviales con textura de migajón arenoso. La vegetación natural está muy modificada, en forma dispersa se encuentran principalmente Acacia farnesiana (L.) Willd., Pithecellobium dulce (Roxb.) Benth. y Crescentia alata Kunth, especies propias del bosque tropical caducifolio (Almazán, 1991). El nuevo taxon se encuentra asociado con plantas características de la vegetación secundaria, como Tithonia diversifolia (Hemsl.) A. Gray, Bidens odorata Cav. y Simsia amplexicaulis (Cav.) Pers.

Distribución: hasta el momento sólo se conoce de México, en el estado de Guerrero.

Por lo característico de sus hojas el nuevo taxon se incluye en Ipomoea sección Leptocallis. En dicha sección cuatro especies presentan raíces tuberosas y otras cuatro especies poseen raíces fibrosas; y por el hecho de presentar raíz fibrosa, I. diegoae se relaciona con las especies I. costellata Torr., I. chamelana J. A. McDonald, I. perpartita McPherson e I. ternifolia Cav. En especial I. ternifolia está más relacionada con el nuevo taxon, ya que se asemeja en las siguientes características: tipo de raíz, forma de las hojas y segmentos, largo de los sépalos y forma de la corola (Cuadro 1).

Ipomoea diegoae difiere de los demás componentes de la sección Leptocallis por presentar hojas más grandes, los segmentos foliares más anchos y en mayor número, los sépalos marcadamente desiguales y más anchos, la corola más grande y de coloración rosa, y finalmente en que los estambres tienen la misma coloración del tubo, desde su base hasta la altura de la garganta.

Etimología: el nombre de la planta se ha asignado como un reconocimiento a la M. en C. Nelly Diego Pérez, por su loable labor en el conocimiento de las plantas mexicanas, quien junto con su equipo de trabajo del laboratorio de Plantas Vasculares de la Facultad de Ciencias en la Universidad Nacional Autónoma de México, está abocada al inventario florístico del estado de Guerrero. 
Cuadro 1. Características comparativas entre I. diegoae y las cuatro especies más relacionadas de la sección Leptocallis. Datos según McDonald (1995) y McPherson (1980).

\begin{tabular}{|c|c|c|c|c|c|}
\hline & I. diegoae & I. costellata & I. chamelana & I. perpartita & I. ternifolia \\
\hline Hábito & $\begin{array}{c}\text { escandente o } \\
\text { postrada }\end{array}$ & $\begin{array}{c}\text { erecta o } \\
\text { escandente }\end{array}$ & escandente & escandente & $\begin{array}{l}\text { escandente o } \\
\text { postrada }\end{array}$ \\
\hline longitud (m) & $0.9-1$ & $0.3-0.7$ & 2 & $2-3$ & $0.5-2$ \\
\hline \multicolumn{6}{|l|}{ Hoja } \\
\hline largo (cm) & $0.9-8.6$ & $1-3.5$ & $0.6-4.2$ & $1-3$ & $1-8$ \\
\hline ancho $(\mathrm{cm})$ & $1.7-9.8$ & $1-4$ & $1-5.2$ & $2-3$ & $1-6$ \\
\hline \multicolumn{6}{|l|}{ Segmentos } \\
\hline número & $14-16$ & $8-10$ & $5-14$ & $3-9$ & $7-11$ \\
\hline $\operatorname{largo}(\mathrm{cm})$ & $1-6.1$ & $0.5-2.8$ & $0.5-4.2$ & $0.1-0.8$ & $0.5-7$ \\
\hline ancho (mm) & $0.3-2.8$ & $1-4$ & $1-3$ & $0.3-0.6$ & $0.2-1.2$ \\
\hline \multicolumn{6}{|l|}{ Pecíolo } \\
\hline $\operatorname{largo}(\mathrm{cm})$ & $0.3-3.6$ & 1.2 & $2.5-3.8$ & $0.2-1.2$ & $1-3$ \\
\hline ancho (mm) & $0.5-0.9$ & $0.3-0.5$ & ca. 0.5 & ca. 1 & $0.2-0.5$ \\
\hline \multicolumn{6}{|l|}{ Cáliz } \\
\hline sépalos & desiguales & iguales & subiguales & $\begin{array}{c}\text { subiguales o } \\
\text { desiguales }\end{array}$ & subiguales \\
\hline $\operatorname{largo}(\mathrm{mm})$ & $7.9-14$ & $3-5$ & $4-5$ & $3.5-7.5$ & $4-14$ \\
\hline ancho (mm) & $2-4.6$ & $1-2$ & 2 & $2-2.5$ & $2-3$ \\
\hline \multicolumn{6}{|l|}{ Corola } \\
\hline forma & $\begin{array}{l}\text { infundibuli- } \\
\text { forme }\end{array}$ & campanulada & $\begin{array}{l}\text { subcampanu- } \\
\text { lada }\end{array}$ & $\begin{array}{l}\text { infundibuli- } \\
\text { forme }\end{array}$ & $\begin{array}{l}\text { infundibulifor- } \\
\text { me o subhipo- } \\
\text { craterimorfa }\end{array}$ \\
\hline $\begin{array}{l}\text { color en } \\
\text { fresco }\end{array}$ & rosa claro & azul, amarillo & amarillo & blanco & $\begin{array}{c}\text { azuloso, morado } \\
\text { y color crema }\end{array}$ \\
\hline $\operatorname{largo}(\mathrm{cm})$ & $4.3-7.2$ & $0.8-1$ & $1.7-2.5$ & $3-4$ & $1.5-4.5$ \\
\hline $\begin{array}{l}\text { diámetro } \\
\text { limbo }(\mathrm{cm})\end{array}$ & $3.9-6.7$ & $0.5-0.8$ & ca. 2 & ca. 2.5 & $3-4$ \\
\hline
\end{tabular}


A continuación se proporciona una clave para la identificación de las especies de la sección Leptocallis del género Ipomoea conocidas para el estado de Guerrero.

1. Hojas basales elípticas o lanceoladas, palmatisectas sólo en los extremos de las ramas maduras; ovario 3 locular I. madrensis

1. Hojas siempre palmatisectas; ovario 2 locular.

2. Raíz tuberosa; hojas sésiles, pecíolo de casi $1 \mathrm{~mm}$ de largo.

3. Hierbas erectas; segmentos foliares filiformes, menores de $1 \mathrm{~mm}$ de ancho

I. capillacea

3. Hierbas decumbentes; segmentos foliares estrechamente elípticos, de más de $1 \mathrm{~mm}$ de ancho

I. plummerae (var. cupulata)

2. Raíz fibrosa; hojas con pecíolo de más de $1 \mathrm{~cm}$ de largo.

4. Flores solitarias, completamente amarillas

I. chamelana

4. Flores en cimas monocasiales o dicasiales simples, rara vez solitarias.

5. Hojas con 7 a 11 segmentos, corola de 1.5 a $4.5 \mathrm{~cm}$ de largo, de color azul o morada, rara vez blanca o de color crema

I. ternifolia (var. ternifolia)

5. Hojas con 14 a 16 segmentos, corola de 4.3 a $7.2 \mathrm{~cm}$ de largo, de color rosa

I. diegoae

\section{AGRADECIMIENTOS}

Se agradece: a la M. en C. Martha Martínez Gordillo, del herbario de la Facultad de Ciencias de la UNAM, por la elaboración de la diagnosis en latín, al Biól. Lucio Lozada del laboratorio de Plantas Vasculares de la Facultad de Ciencias, por sus sugerencias y comentarios, a Jorge Martínez Domínguez y Luis Francisco Barcelata por la realización de la ilustración, y a Israel Noriega por su ayuda en la captura para la elaboración del cuadro.

\section{LITERATURA CITADA}

Almazán, J. A. 1991. Estudio florístico-ecológico de la maleza de los campos de cultivo de riego, en el valle de Iguala, Gro. Tesis de maestría. Facultad de Ciencias, Universidad Nacional Autónoma de México. México, D.F. 137 pp.

Austin, D. F. 1979. An infrageneric classification for Ipomoea (Convolvulaceae). Taxon 28(4): 359-361.

Austin, D. F. 1980. Additional comments on infrageneric taxa in Ipomoea (Convolvulaceae). Taxon 29(4): 501-502. 
House, H. D. 1908 . The North American species of the genus Ipomoea. Ann. New York Acad. Sci. 18(6): 181-263.

Matuda, E. 1963. El género Ipomoea en México I. Anales Inst. Biol. Univ. Nac. México 34: 85-145.

McDonald, J. A. 1995. Revision of Ipomoea section Leptocallis (Convolvulaceae). Harvard Papers in Botany 1(6): 97-122.

McPherson, G. D. 1980. Eight new species of Ipomoea and Quamoclit from Mexico. Contr. Univ. Mich. Herb. 14: 85-97. 